\title{
Plasma guiding using a chaperone pre-pulse for stable wakefield generation
}

Devki Nandan Gupta

University of Delhi

Samuel Robert Yoffe ( $\nabla$ sam.yoffe@strath.ac.uk)

University of Strathclyde

Arohi Jain

University of Delhi

Bernhard Ersfeld

University of Strathclyde

Dino Anthony Jaroszynski

University of Strathclyde

\section{Research Article}

Keywords: laser-plasma, ultra-compact accelerators, VHEE, LWFA, ICLs, sub-picosecond

Posted Date: July 12th, 2021

DOl: https://doi.org/10.21203/rs.3.rs-679840/v1

License: (c) (1) This work is licensed under a Creative Commons Attribution 4.0 International License.

Read Full License 


\title{
Plasma guiding using a chaperone pre-pulse for stable wakefield generation
}

\author{
D. N. Gupta ${ }^{1,+}$, S. R. Yoffe ${ }^{2, \dagger,+}$ A. Jain ${ }^{1,+}$, B. Ersfeld ${ }^{2}$, and D. A. Jaroszynski ${ }^{2, *}$ \\ ${ }^{1}$ Department of Physics and Astrophysics, University of Delhi, Delhi 110 007, India \\ ${ }^{2}$ Department of Physics, SUPA \& University of Strathclyde, Glasgow G4 0NG, United Kingdom \\ Corresponding author: sam.yoffe@strath.ac.uk \\ *Corresponding author: d.a.jaroszynski@strath.ac.uk \\ +These authors contributed equally to this work
}

\begin{abstract}
Achieving high quality electron beams in laser wakefield accelerators requires stable guiding of the intense driving laser pulse, which is challenging because of mode mismatching due to relativistic self-focusing. Here we show how an intense pre-pulse can be used to prepare the phase-space distribution of plasma electrons encountered by a trailing laser pulse so that it produces its own well-matched guiding channel, while minimising wakefield evolution. Controlling the propagation of high intensity laser pulses is an essential step in developing useful wakefield accelerators and compact radiation sources.
\end{abstract}

\section{Introduction}

The laser-plasma accelerator ${ }^{1-4}$ has advanced to the stage where control of beam parameters ${ }^{5-11}$ and stability are the main development challenges. Achieving high quality electron beams in laser wakefield accelerators requires stable guiding of the driving laser pulse, ${ }^{12-20}$ which is challenging because of mode mismatching due to relativistic self-focusing. Here we show how an intense pre-pulse can be used to prepare the phase-space distribution of plasma electrons encountered by a trailing driver pulse so that it produces its own well-matched guiding channel that minimises wakefield evolution. This is distinct from the pre-formed plasma channel, which requires excellent alignment between channel and driver pulse ${ }^{21,22}$. Controlling high intensity driver pulses is an essential step in developing useful wakefield accelerators and compact radiation sources ${ }^{23-25}$. Stable channels are necessary for developing next generation compact plasma undulators for compact synchrotron sources ${ }^{26,27}$ and free-electron lasers ${ }^{28,29}$. Wide availability of ultra-compact accelerators and radiation sources could transform the way science is done $\mathrm{e}^{23,30}$.

The laser wakefield accelerator (LWFA) ${ }^{1}$ utilizes the ponderomotive force of an intense, ultra-short duration driver pulse to separate electrons from ions. This produces an ultra-high electric field that can exceed $100 \mathrm{GV} / \mathrm{m}$, which is several orders of magnitude larger than possible in conventional accelerators. At high laser intensities, displaced electrons follow trajectories that define a thin sheath surrounding the ion-filled plasma bubble. Electrons converging at the rear of the bubble that have a velocity exceeding the wake velocity can be injected into the bubble and rapidly accelerated to high energies. LWFAs have produced high energy electron beams ${ }^{5,6,11}$ with excellent properties ${ }^{7-10}$. They also form the basis of compact all-optical sources of brilliant X-ray ${ }^{26}$ and gamma-ray ${ }^{27}$ photons that have potential applications including spectroscopy and imaging for material sciences, warm dense matter studies for laboratory astrophysics and fusion, and for the biology and health sciences ${ }^{31-33}$. Ultra-short pulse LWFAs are being investigated as drivers of synchrotron sources ${ }^{24}$, free-electron lasers ${ }^{23-25}$ and ion channel lasers (ICLs) ${ }^{28,29}$, and for very high energy electron (VHEE) radiotherapy ${ }^{34-36}$, pulsed radiolysis ${ }^{37}$ and medical imaging ${ }^{32,33}$. Many applications require high-quality electron bunch properties, such as low emittance, ultra-short duration and high peak current. It is well known that excellent bunch properties depend on maintaining field uniformity ${ }^{23,30}$, and that bubble evolution directly influences self-injection ${ }^{10,38,39}$. Laser $^{40}$ and plasma ${ }^{38}$ variations drive bubble evolution, resulting in time-varying electrostatic fields that impact electron bunch quality ${ }^{41}$. To mitigate these variations and ensure persistent acceleration, stable guiding of the driver pulse is necessary.

Several methods of guiding intense laser pulses have been developed. Plasma waveguides can be preformed using electrical discharges in capillaries ${ }^{12-14}$ or heating plasma using long- (nanosecond) ${ }^{15-17}$ or short-duration (sub-picosecond) ${ }^{18-20}$ laser pulses. Matching a waveguide and controlling guiding is challenging at very high intensities $\left(I>10^{18} \mathrm{~W} / \mathrm{cm}^{2}\right.$ at wavelength $\lambda=800 \mathrm{~nm})$. When the power is higher than the critical power, $P>17.4\left(\lambda_{p} / \lambda\right)^{2} \mathrm{GW}^{42}$, the preformed plasma waveguide properties are modified due to relativistic changes to the local refractive index, which gives rise to self-focusing ${ }^{43-46}$. (The plasma wavelength is defined as $\lambda_{p}=2 \pi c \sqrt{m_{e} \varepsilon_{0} /\left(n_{0} e^{2}\right)}$, with $c\left(\varepsilon_{0}\right)$ the speed of light (permittivity) in vacuum, $e$ the 
fundamental charge, $m_{e}$ the mass of the electron, and $n_{0}$ the plasma electron number density.) Mode mismatch results in significant variation of the driver pulse diameter, known as scalloping. Moreover, misalignment or large beam diameters can cause catastrophic damage to preformed capillaries ${ }^{11,13,46}$ and severely reduce transmission ${ }^{46}$.

Many applications require low density plasma, and thus relatively large channel diameters and large matched laser spot sizes. While guiding of high-power $(850 \mathrm{TW})$ pulses in a preformed low density plasma channel has been demonstrated ${ }^{11}$ in an $800 \mu \mathrm{m}$-diameter capillary with $60 \mu \mathrm{m}$ laser focal spot, the authors note that at this density and capillary diameter "the capillary discharge waveguide produces a channel that is not deep enough to sufficiently confine the laser pulse" and, therefore, an additional nanosecond heater laser pulse is used to deepen the waveguide to enable guiding over 15 Rayleigh lengths.

In this article, we present a unique double-pulse scheme that creates a stable plasma channel waveguide intrinsically aligned with the trailing driver pulse. The plasma momentum distribution and electrostatic field are prepared by an intense, shortduration pre-pulse that precedes the arrival of a second, co-propagating pulse. The time delay between the two pulses is chosen to be slightly less than the plasma period, $t_{d}<\lambda_{p} / c$ and the moderately intense leading, or chaperone, pre-pulse excites a weakly-nonlinear plasma wave that is below the threshold for wavebreaking and self-injection. The pre-pulse does not directly produce a guiding structure for the trailing driver pulse, but rather its wake prepares the plasma by driving converging streams of electrons that are subsequently deflected by the ponderomotive force of the (trailing) driver pulse to produce a narrow, well-defined parabolic plasma channel. This acts as a self-aligned waveguide accompanying the driver over extended propagation lengths. The driver pulse initially undergoes relativistic self-focusing so that it matches the narrow density channel, which ensures high coupling efficiency. The chaperone pre-pulse stimulates guiding of the driver pulse, providing control over its evolution and enhancing the stability of the resulting wakefield. This can also be used to suppress spontaneous self-injection and provide better controlled electron bunch properties.

\section{Results}

Particle-in-cell (PIC) simulations have been performed (see Methods) to evaluate the effect of the leading pre-pulse. We compare the double pulse configuration to the case without the pre-pulse, as well as a single pulse containing the same total energy as the driver and pre-pulse combined.

As described above, the purpose of the pre-pulse is not to directly produce a plasma channel to guide the driver pulse, but to prepare the plasma to facilitate the production of a channel by the driver pulse (see Supplementary Fig. S1). The delay is fine-tuned to ensure that electrons displaced by the pre-pulse interact with the driver pulse as they converge at the back of the pre-pulse wake, where they are deflected to produce a narrow, well-defined parabolic density channel [Fig. 1(b)] that guides the driver pulse. In contrast, the density modulation produced by a single driver pulse, as illustrated in Fig. 1(a), shows the familiar wake without evidence of a channel structure. In Fig. 1(c), the electron density shows no structure to guide the single driving laser (red), whereas in Fig. 1(d) a waveguide stimulated by the pre-pulse (green) is evident in the region of the driver pulse (red). When guided, the driver pulse maintains a high peak intensity and a narrower beam waist over a long distance. This demonstrates the formation of a channel that is capable of guiding an intense laser pulse in plasma with density $7.17 \times 10^{17} \mathrm{~cm}^{-3}$. A similar plasma channel can be created in $1 \times 10^{17} \mathrm{~cm}^{-3}$ plasma, as shown in Figs. 1(e) and (f) with initial $a_{0}=a_{1}=2.5$. Due to the increased resources required to perform these simulations at lower density, the latter has not been fully optimized. However, they clearly demonstrate guiding channel formation at lower densities.

Figure 2 presents the transverse density profile (averaged over half a laser wavelength) at the position of maximum intensity of the driver pulse for the cases (a) without the pre-pulse; (b) with the pre-pulse; and (c) a single higher-intensity driver pulse. In the single pulse cases, Figs. 2(a) and (c), the density is peaked on the laser axis, and no channel structure is observed. In contrast, Fig. 2(b) clearly demonstrates the production of a density channel with steep walls and reduced density at the center, which exists as the laser pulse propagates from around $2 \mathrm{~mm}$ to $12 \mathrm{~mm}$. The solid curve shows the channel radius, $r_{c}$, for a parabolic channel of the form $n_{e}=n_{\min }\left(1+r^{2} / r_{c}^{2}\right)$, where $n_{\min }$ is the density at the center of the channel. The lineouts (cross-section) of the transverse density profile at $2.7 \mathrm{~mm}$ (dashed), $8 \mathrm{~mm}$ (dot-dashed), and $12 \mathrm{~mm}$ (dot-dot-dashed) are shown in panels Fig. 2(d,e,f), along with the corresponding parabolic profile in orange. We observe that the channel is well described by a parabola and that the structure accompanies the driver pulse over an extended length.

The production of a co-moving parabolic density channel is not sufficient to claim that the driver pulse is guided. Figure 3(a) shows the temporal evolution of the amplitude with and without the pre-pulse, and for vacuum propagation. The driver pulse alone is observed to self-focus in the plasma before diffracting. However, a stable amplitude is observed with a pre-pulse. Similarly, the driver pulse waist is stable when the pre-pulse is used [Fig. 3(b)]. Stable driver pulse amplitude and waist indicate a matched guided pulse, confirming that the parabolic density channel acts as a well-matched waveguide. The matched laser spot size ${ }^{13}$ for the instantaneous plasma channel density and normalized laser amplitude at $7 \mathrm{~mm}$ is $W_{M}=13.9 \mu \mathrm{m}$, while the value of the laser spot is $r_{l}=14.9 \mu \mathrm{m}$. This is in good agreement, considering that it does not account for relativistic effects or the electron momentum distribution generated by the pre-pulse. Data presented in Fig. 3 for the single pulse LWFA (-- -) originally appeared in Yoffe et al. ${ }^{47}$. 
$\mathbf{a}$
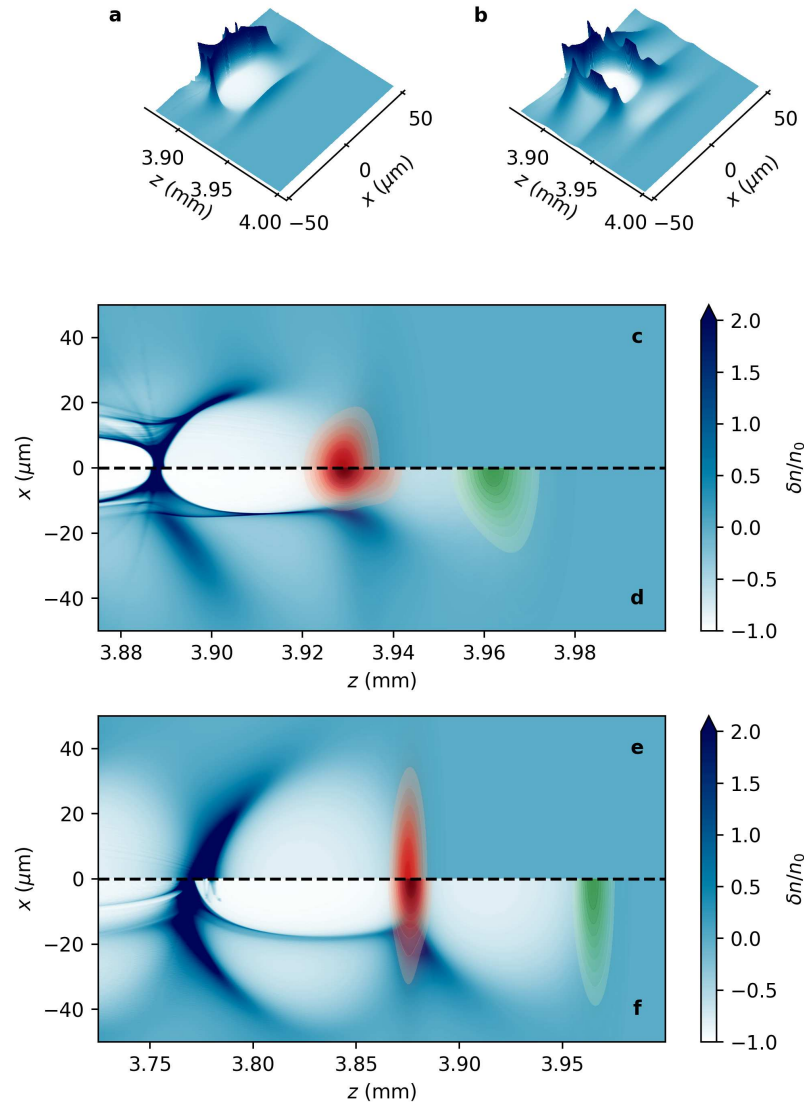

$\stackrel{2}{\frac{1}{5}}$ . (5)

Figure 1. Simulation results demonstrate the formation of the guiding channel stimulated by the pre-pulse. The electron density $\left(n_{e}\right)$ modulation $\delta n / n_{0}=\left(n_{e}-n_{0}\right) / n_{0}$ (with $n_{0}=7.17 \times 10^{17} \mathrm{~cm}^{-3}$ ) visualized (a) without and (b) with a pre-pulse. (Note that the vertical axis has been truncated at $\delta n / n_{0}=2$ for clarity.) The electron density and the driver pulse intensity (red) are shown in part (c) without, and in part (d) with, the pre-pulse laser (green). Similar production of a density channel in lower density plasma $\left(n_{0}=1 \times 10^{17} \mathrm{~cm}^{-3}\right)$ is illustrated by parts (e) and (f), without and with the pre-pulse, respectively. Parts (a)-(f) use the same colour scale.

The discontinuities observed for the single pulse cases [Figs. 2(a),(c) and 3(a),(b)] are artefacts due to sudden jumps in the position of the intensity maximum. As the rear of the driver pulse is focused, it produces a second peak that grows to attain the highest intensity. This is illustrated in Fig. 2(g) for the high-intensity single driver pulse.

A consequence of guiding the intense driver pulse is a significant improvement of the stability of the plasma wave: the bubble maintains a consistent shape and velocity, minimizing time-dependent fields that would otherwise lead to increases in energy spread and emittance ${ }^{41}$. Figure 4 shows the time evolution of the on-axis longitudinal electric field, $E_{z}$. Figures $4($ a) and $4(\mathrm{c})$ show the field for the single driver pulse cases, where the center (dotted line) and back of the bubble are observed to fluctuate significantly. In Fig. 4(a) we also observe that the longitudinal field is only strong over a short propagation distance. However, in Fig. 4(b) with the chaperone pre-pulse, the strong field segment and the bubble velocity are much more stable.

Figure 5(a) shows the evolution of the velocity of the back of the bubble (defined as the zero-crossing of the longitudinal electric field) with the shaded region enlarged in Fig. 5(b). The velocity of the dephasing point is presented in Fig. 5(c). Data presented in Fig. 5(c) for the single pulse LWFA (- - ) originally appeared in Yoffe et al. ${ }^{47}$. The (dimensionless) bubble velocity, $\beta_{b}$, and dephasing point velocity, $\beta_{d}$, are observed to vary significantly for the two cases without the pre-pulse, becoming superluminal. The presence of the pre-pulse and the stimulated guiding of the driver pulse stabilizes both the laser evolution (such as its group velocity ${ }^{47}$ ) and the wakefield it generates (including the bubble and dephasing point velocities). The increased stability of the wake using a double pulse is ideal for high-quality laser wakefield accelerators (LWFAs), and also for applications of ion channels for ion channel lasers (ICLs) ${ }^{29}$, which require the radiating electron bunch to stay at the dephasing point to minimise 

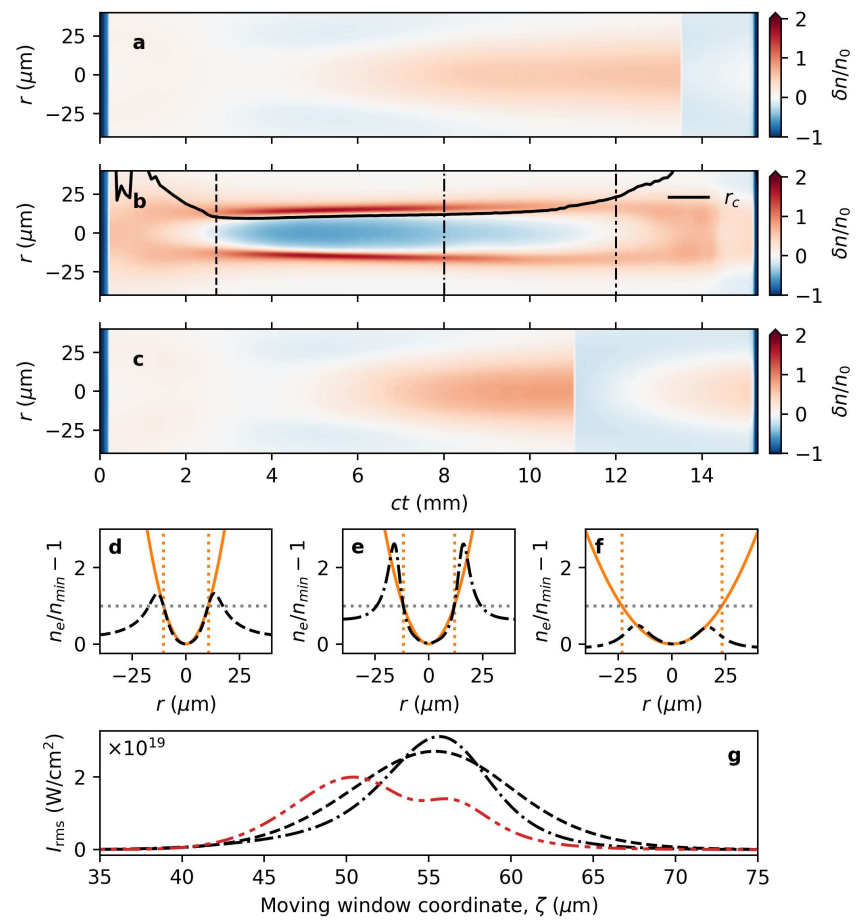

Figure 2. Evolution of the transverse electron density profile at the laser peak. Evolution of the transverse electron density profile, determined at the position of peak intensity of the driver pulse, for the (a) single driver pulse; (b) pre-pulse and driver pulse; and (c) higher-intensity single driver pulse configurations. Panel (b) demonstrates the production of a persistent plasma channel, with steep walls and a low density core. The maximum channel height at the position of peak laser intensity is $\delta n / n_{0}=2$. Lineouts of the density profile at $c t=2.7 \mathrm{~mm}(--), c t=8 \mathrm{~mm}(\cdot-)$, and $c t=12 \mathrm{~mm}(\cdot-)$ from part (b) are shown in panels (d)-(f). For the double pulse case, the corresponding parabolic channel profile is plotted in orange, indicating that a parabolic density channel is produced and maintained over a significant distance. The measured channel radius is (d) $r_{c}=10.4 \mu \mathrm{m},(\mathrm{e}) r_{c}=11.8 \mu \mathrm{m}$, and (f) $r_{c}=23.3 \mu \mathrm{m}$. Part (g) shows the on-axis intensity (as a function of the moving window coordinate, $\zeta=z-c t$ ) for the higher-intensity single pulse at the three positions, as above, illustrating the development of a second peak that becomes the maximum, causing the discontinuity observed in parts (a) and (c).

energy variation. Matching the electron bunch velocity to that of the dephasing point is only feasible if the latter varies slowly, which is achieved with the pre-pulse. External injection of electron bunches into a laser wakefield bubble at the dephasing point is possible, but the laser evolution usually results in the electrons not remaining at the dephasing point for long. In contrast, with the pre-pulse, the velocity of the dephasing point varies slowly, so that electron bunches injected with the correct velocity and phase will remain near the dephasing point for an extended period.

\section{Conclusions}

We have demonstrated how the introduction of a relatively low-intensity chaperone pre-pulse in the LWFA can stimulate the production of a guiding plasma channel by a trailing driver pulse. For the parameters investigated here, we find that a delay of $0.85 \lambda_{p} / c$ results in a parabolic density channel that acts as a waveguide for a focused high-intensity $\left(I_{\mathrm{rms}}>4.5 \times 10^{19} \mathrm{~W} / \mathrm{cm}^{2}\right)$ $430 \mathrm{TW}$ pulse over nearly a centimetre $(\approx 10$ Rayleigh lengths for the focused pulse in vacuum) in low density plasma, where it is usually difficult to produce suitable plasma waveguides ${ }^{11}$. The scheme presented here does not require alignment or synchronization, which are usually challenging when using preformed plasma waveguides. The driver pulse energy coupling efficiency into the channel at $2.7 \mathrm{~mm}$ is $98.7 \%$, with an overall guiding efficiency of $89.3 \%$ observed when the laser propagates from $2.7 \mathrm{~mm}$ to $12 \mathrm{~mm}$ (including the loss of laser pulse energy to create the channel and wake). Guiding could be further improved by optimization.

It is important that the pre-pulse is well matched to the plasma so that it persists over the required interaction length. For the results obtained here, we found that the best channel and guiding are observed when the pre-pulse amplitude $a_{0}>1.7$. To extend the scheme to lower densities, while matching the pre-pulse parameters for stable propagation, it will be necessary to 

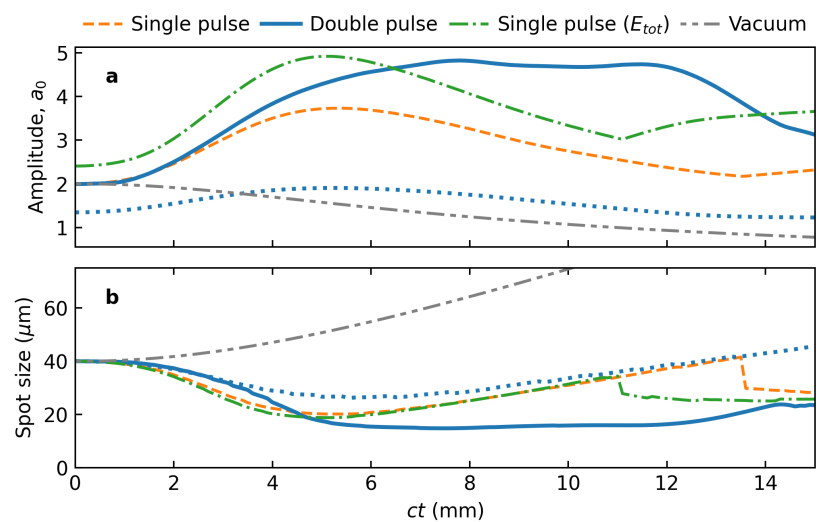

Figure 3. Comparison of driver pulse evolution for the single and double pulse cases. Evolution of the driver pulse (a) amplitude and (b) waist, for the single driver pulse (-- -), double pulse (-), and higher-intensity single driver pulse $(\cdot-)$ cases. A region of stable driver pulse guiding is identified for the double pulse case between $5 \mathrm{~mm}$ and $12 \mathrm{~mm}$. Vacuum propagation for the (initial) driver pulse $(\cdots-)$ is shown for comparison, along with the evolution of the pre-pulse $(\cdots)$. Data for the single pulse LWFA (---) originally appeared in Yoffe et al. ${ }^{47}$.
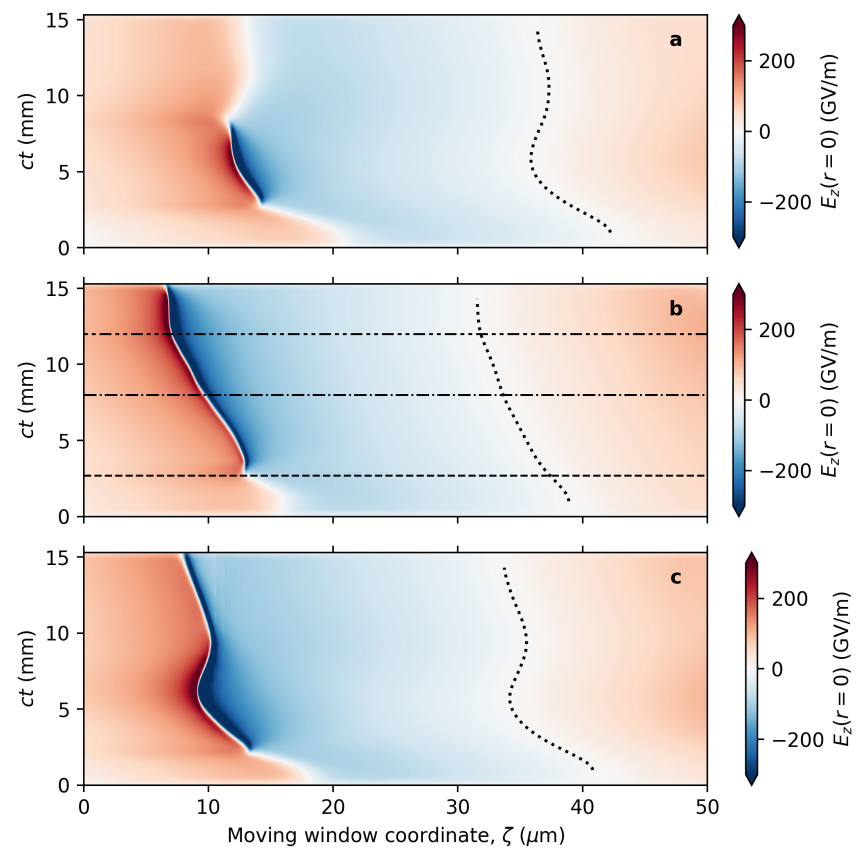

Figure 4. Evolution of the longitudinal electric field. Temporal evolution of the on-axis longitudinal electric field for the (a) single pulse; (b) double pulse; and (c) higher-intensity single pulse configurations, shown in the moving window, $\zeta=z-c t$ (a vertical line would represent motion at $c$ ). Horizontal lines indicate the propagation distances $2.7 \mathrm{~mm}, 8 \mathrm{~mm}$ and $12 \mathrm{~mm}$ highlighted and discussed in Fig. 2. Dotted lines indicate the position of the dephasing point.

increase its width and power to remain above the critical power to excite enough electrons to assemble a sufficiently dense channel to guide the driver pulse. The channel must also be wider to ensure that the group velocity of the driver pulse is matched to that of the pre-pulse.

The stabilizing effect of the chaperone pre-pulse significantly improves the overall stability of the plasma wake. The doublepulse configuration should contribute to the development of controllable LWFAs for high-energy, high-quality electron bunches for a wide range of applications, such as drivers of secondary radiation sources. We also note that the stability of the ion 

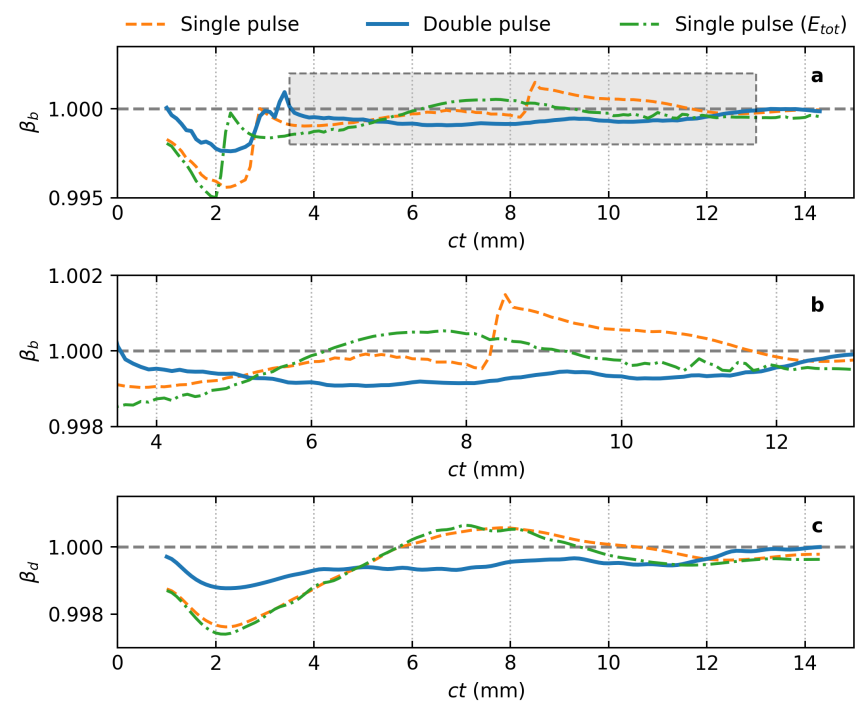

Figure 5. Evolution of the bubble velocity. Part (a) shows the variation of the (normalized) velocity of the back of the bubble, $\beta_{b}$ for the single pulse (---), double pulse (-), and higher-intensity single pulse (- - ) configurations, with the highlighted region expanded in part (b). Part (c) shows the variation of the (normalized) velocity of the dephasing point at the center of the bubble, $\beta_{d}$. Data presented in Fig. 5(c) for the single pulse LWFA (-- -) originally appeared in Yoffe et al. ${ }^{47}$.

cavity, driver pulse amplitude and plasma density can be used to control the velocity of the dephasing point, making this a very attractive ion channel for an ICL or plasma wiggler. To design an experiment to verify chaperone guiding, similar results are obtained using parallel polarizations for the pre-pulse and driver pulse.

\section{Methods}

Computer simulations are performed using the open source code FBPIC ${ }^{48}$, which is a quasi-3D PIC code that uses cylindrical geometry and azimuthal modal decomposition to represent the 3D domain. This significantly reduces the computational resources required, especially for large domains, while retaining important 3D effects. The spectral solver used by the code reduces numerical artifacts, such as noise, dispersion and numerical Cherenkov radiation. Simulations have been performed for various laser intensities for single and double laser pulses, using a moving window of size $z \times r=156.25 \lambda_{0} \times 125 \lambda_{0}$ with $3126 \times 1000$ grid points propagating at the speed of light. From the geometry of the laser and plasma, it is expected that only azimuthal modes $m=0,1$ will be important. The simulations presented here use azimuthal modes $m=0,1,2$ with $(3,2,12)$ particles per cell in $(z, r, \theta)$, but results are almost identical to the case using two modes and fewer particles per cell.

We first consider the wakefield generated by a single Gaussian-profile driver pulse with an initial normalized amplitude $a_{1}=2$, which is defined from the electric field, $\boldsymbol{E}$, as $a=e \boldsymbol{\lambda}|\boldsymbol{E}| /\left(2 \pi m_{e} c^{2}\right)$, where $\lambda_{1}=800 \mathrm{~nm}$ is the peak laser wavelength (pulse intensity $I_{\mathrm{rms}}=8.55 \times 10^{18} \mathrm{~W} / \mathrm{cm}^{2}$ ). The driver pulse has full-width at half-maximum (FWHM) intensity duration $\tau_{1}=45 \mathrm{fs}$ and focal spot size (at $1 / e^{2}$ intensity) $r_{1}=40 \mu \mathrm{m}$ (energy $10.29 \mathrm{~J}$ ). Simulations are then repeated adding a prepulse (with the same wavelength, duration and spot size as above) but with initial normalized amplitude $a_{0}=1.35$ (intensity $I_{\mathrm{rms}}=3.90 \times 10^{18} \mathrm{~W} / \mathrm{cm}^{2}$, energy $\left.4.69 \mathrm{~J}\right)$. Plasma waves are resonantly excited ${ }^{49}$ when the delay length is equal to the plasma wavelength, $\lambda_{p}$. However, here we choose a delay $c t_{d}=0.85 \lambda_{p}$ so that the position of the driver pulse is in front of the density maximum of the (unperturbed) pre-pulse wake. To clearly distinguish the pre-pulse from the driver pulse, the polarizations are chosen to be orthogonal. However, similar results are obtained for pulses with the same polarization. Finally, a third case is considered using a single Gaussian-profile driver pulse containing the total energy of the driver and pre-pulse from the double pulse case above, $E_{\mathrm{tot}}=14.98 \mathrm{~J}$ with an initial normalized amplitude $a_{1}=2.413$ (intensity $I_{\mathrm{rms}}=1.24 \times 10^{19} \mathrm{~W} / \mathrm{cm}^{2}$ ). The plasma is taken to be a $15 \mathrm{~mm}$ flat-top plateau with electron number density $n_{0}=7.17 \times 10^{17} \mathrm{~cm}^{-3}$ and $100 \mu \mathrm{m}$ sine-profile ramps. Laser pulses are focused at the top of the plasma density upramp. These parameters are chosen for a proof-of-principle demonstration, which could be further optimized.

As a guide to finding a starting point for the delay optimization, we use a nonlinear $1 \mathrm{D}$ theory ${ }^{43,50}$ with the plasma and pre-pulse parameters. We consider a laser pulse propagating along the $z$-axis with Gaussian profile defined by $a^{2}(\zeta)=a_{0}^{2} \exp \left(-\zeta^{2} /\left(c \tau_{0}\right)^{2}\right)$, where $\zeta=z-v_{g} t$ with $v_{g}$ the laser group velocity and $\tau_{0}$ is the laser pulse duration. The normalized wake potential 
$\phi=e \Phi /\left(m_{e} c^{2}\right)$ is obtained by numerically solving the second-order nonlinear differential equation for the nonlinear regime:

$$
\frac{\partial^{2} \phi}{\partial \zeta^{2}}=k_{p}^{2} \gamma_{p}^{2}\left[\beta_{p}\left(1-\frac{1+a^{2}}{\gamma_{p}^{2}(1+\phi)^{2}}\right)^{-1 / 2}-1\right],
$$

where $\gamma_{p}=\left(1-\beta_{p}^{2}\right)^{-1 / 2}$ is the relativistic Lorentz factor associated with the phase velocity of the plasma wave $v_{p}$, and $\beta_{p}=v_{p} / c$. The plasma wavenumber is defined as $k_{p}=\omega_{p} / v_{p}$ with the plasma frequency given by $\omega_{p}=\sqrt{n_{0} e^{2} /\left(m_{e} \varepsilon_{0}\right)}$. The longitudinal electric field, $E_{z}$, and electron density modulation, $\delta n / n_{0}$ are given as:

$$
\begin{aligned}
& \frac{E_{z}}{E_{0}}=-\frac{1}{k_{p}} \frac{\partial \phi}{\partial \zeta} \\
& \frac{\delta n}{n_{0}}=\frac{1}{k_{p}^{2}} \frac{\partial^{2} \phi}{\partial \zeta^{2}},
\end{aligned}
$$

where $E_{0}=m_{e} c \omega_{p} / e$ is the wave breaking field that corresponds to the maximum possible electric field of the plasma wave. The above equations are solved to estimate the plasma response to the pre-pulse, and the corresponding numerical results are illustrated in the Supplementary Fig. S2, where the position of the peak of the driver pulse is chosen to be $0.85 \lambda_{p}$ from the position of pre-pulse. As an initial value to begin optimization we suggest choosing the peak of the driver pulse positioned ahead of the density peak driven by the pre-pulse (see Supplementary Information for details).

\section{References}

1. Tajima, T. \& Dawson, J. M. Laser Electron Accelerator. Phys. Rev. Lett. 43, 267-270 (1979).

2. Mangles, S. P. D. et al. Monoenergetic beams of relativistic electrons from intense laser-plasma interactions. Nature 431, 535-538 (2004).

3. Geddes, C. G. R. et al. High-quality electron beams from a laser wakefield accelerator using plasma-channel guiding. Nature 431, 538-541 (2004).

4. Faure, J. et al. A laser-plasma accelerator producing monoenergetic electron beams. Nature 431, 541-544 (2004).

5. Leemans, W. P. et al. GeV electron beams from a centimetre-scale accelerator. Nature Physics 2, 696-699 (2006).

6. Leemans, W. P. et al. Multi-GeV Electron Beams from Capillary-Discharge-Guided Subpetawatt Laser Pulses in the Self-Trapping Regime. Physical Review Letters 113, 245002 (2014).

7. Wiggins, S. M. et al. High quality electron beams from a laser wakefield accelerator. Plasma Physics and Controlled Fusion 52, 124032 (2010).

8. Brunetti, E. et al. Low emittance, high brilliance relativistic electron beams from a laser-plasma accelerator. Physical Review Letters 105, 215007 (2010).

9. Lundh, O. et al. Few femtosecond, few kiloampere electron bunch produced by a laser-plasma accelerator. Nature Physics 7, 219-222 (2011).

10. Islam, M. R. et al. Near-threshold electron injection in the laser-plasma wakefield accelerator leading to femtosecond bunches. New Journal of Physics 17, 093033 (2015).

11. Gonsalves, A. et al. Petawatt laser guiding and electron beam acceleration to $8 \mathrm{GeV}$ in a laser-heated capillary discharge waveguide. Physical Review Letters 122, 084801 (2019).

12. Ehrlich, Y. et al. Guiding of high intensity laser pulses in straight and curved plasma channel experiments. Phys. Rev. Lett. 77, 4186-4189 (1996).

13. Butler, A., Spence, D. J. \& Hooker, S. M. Guiding of high-intensity laser pulses with a hydrogen-filled capillary discharge waveguide. Phys. Rev. Lett. 89, 185003 (2002).

14. Abuazoum, S. et al. Linearly tapered discharge capillary waveguides as a medium for a laser plasma wakefield accelerator. Applied Physics Letters 100, 014106 (2012).

15. Durfee, C. G. \& Milchberg, H. M. Light pipe for high intensity laser pulses. Phys. Rev. Lett. 71, 2409-2412 (1993).

16. Volfbeyn, P., Esarey, E. \& Leemans, W. P. Guiding of laser pulses in plasma channels created by the ignitor-heater technique. Physics of Plasmas 6, 2269-2277 (1999). 
17. Xiao, Y.-F. et al. Efficient generation of extended plasma waveguides with the axicon ignitor-heater scheme. Physics of Plasmas 11, L21-L24 (2004).

18. Kumarappan, V., Kim, K. Y. \& Milchberg, H. M. Guiding of intense laser pulses in plasma waveguides produced from efficient, femtosecond end-pumped heating of clustered gases. Phys. Rev. Lett. 94, 205004 (2005).

19. Lemos, N. et al. Effects of laser polarization in the expansion of plasma waveguides. Physics of Plasmas 20, 103109 (2013).

20. Lemos, N. et al. Plasma expansion into a waveguide created by a linearly polarized femtosecond laser pulse. Physics of Plasmas 20, 063102 (2013).

21. Ibbotson, T. P. A. et al. Investigation of the role of plasma channels as waveguides for laser-wakefield accelerators. New Journal of Physics 12, 045008 (2010).

22. Hooker, S. M. Developments in laser-driven plasma accelerators. Nature Photonics 7, 775 (2013).

23. Jaroszynski, D. A. \& Vieux, G. Coherent Radiation Sources Based on Laser Plasma Accelerators. AIP Conference Proceedings 647, 902-914 (2002).

24. Schlenvoigt, H.-P. et al. A compact synchrotron radiation source driven by a laser-plasma wakefield accelerator. Nature Physics 4, 130-133 (2008).

25. Anania, M.-P. et al. An ultrashort pulse ultra-violet radiation undulator source driven by a laser plasma wakefield accelerator. Applied Physics Letters 104, 264102 (2014).

26. Rousse, A. et al. Production of a keV X-Ray Beam from Synchrotron Radiation in Relativistic Laser-Plasma Interaction. Phys. Rev. Lett. 93, 135005 (2004).

27. Cipiccia, S. et al. Gamma-rays from harmonically resonant betatron oscillations in a plasma wake. Nature Physics $\mathbf{7}$, 867-871 (2011).

28. Whittum, D. H., Sessler, A. M. \& Dawson, J. M. Ion-channel laser. Phys. Rev. Lett. 64, 2511-2514 (1990).

29. Ersfeld, B. et al. The ion channel free-electron laser with varying betatron amplitude. New Journal of Physics 16, 093025 (2014).

30. Jaroszynski, D. et al. Radiation sources based on laser-plasma interactions. Philosophical Transactions of the Royal Society A: Mathematical, Physical and Engineering Sciences 364, 689-710 (2006).

31. Wenz, J. et al. Quantitative x-ray phase-contrast microtomography from a compact laser-driven betatron source. Nature Communications 6, 7568 (2015).

32. Cole, J. M. et al. Laser-wakefield accelerators as hard x-ray sources for $3 \mathrm{~d}$ medical imaging of human bone. Scientific Reports 5, 13244 (2015).

33. Cole, J. M. et al. High-resolution $\mu \mathrm{ct}$ of a mouse embryo using a compact laser-driven x-ray betatron source. Proceedings of the National Academy of Sciences 115, 6335-6340 (2018).

34. Fuchs, T. et al. Treatment planning for laser-accelerated very-high energy electrons. Physics in Medicine and Biology 54, 3315-3328 (2009).

35. Subiel, A. et al. Challenges of dosimetry of ultra-short pulsed very high energy electron beams. Physica Medica 42, 327-331 (2017).

36. Kokurewicz, K. et al. Focused very high-energy electron beams as a novel radiotherapy modality for producing high-dose volumetric elements. Scientific Reports 9, 10837 (2019).

37. Yang, X., Brunetti, E. \& Jaroszynski, D. A. High-energy coherent terahertz radiation emitted by wide-angle electron beams from a laser-wakefield accelerator. New Journal of Physics 20, 043046 (2018).

38. Tooley, M. P. et al. Towards Attosecond High-Energy Electron Bunches: Controlling Self-Injection in Laser-Wakefield Accelerators Through Plasma-Density Modulation. Phys. Rev. Lett. 119, 044801 (2017).

39. Gopal, K. \& Gupta, D. N. Optimization and control of electron beams from laser wakefield accelerations using asymmetric laser pulses. Physics of Plasmas 24, 103101 (2017).

40. Benedetti, C., Schroeder, C. B., Esarey, E. \& Leemans, W. P. Quasi-matched propagation of ultra-short, intense laser pulses in plasma channels. Physics of Plasmas 19, 053101 (2012).

41. Khachatryan, A. G., Irman, A., van Goor, F. A. \& Boller, K.-J. Femtosecond electron-bunch dynamics in laser wakefields and vacuum. Phys. Rev. ST Accel. Beams 10, 121301 (2007). 
42. Esarey, E., Sprangle, P., Krall, J. \& Ting, A. Self-focusing and guiding of short laser pulses in ionizing gases and plasmas. IEEE Journal of Quantum Electronics 33, 1879-1914 (1997).

43. Sprangle, P., Esarey, E. \& Ting, A. Nonlinear theory of intense laser-plasma interactions. Phys. Rev. Lett. 64, 2011-2014 (1990).

44. Gupta, D. N., Hur, M. S., Hwang, I., Suk, H. \& Sharma, A. K. Plasma density ramp for relativistic self-focusing of an intense laser. J. Opt. Soc. Am. B 24, 1155-1159 (2007).

45. Ralph, J. E. et al. Self-guiding of ultrashort, relativistically intense laser pulses through underdense plasmas in the blowout regime. Phys. Rev. Lett. 102, 175003 (2009).

46. Lemos, N. et al. Guiding of laser pulses in plasma waveguides created by linearly-polarized femtosecond laser pulses. Scientific Reports 8, 3165 (2018).

47. Yoffe, S. R. et al. Controlling the group velocity of an intense laser pulse using a pre-pulse. In Jaroszynski, D. A. \& Hur, M. (eds.) Relativistic Plasma Waves and Particle Beams as Coherent and Incoherent Radiation Sources IV, vol. 11778, 8 17. International Society for Optics and Photonics (SPIE, 2021).

48. Lehe, R., Kirchen, M., Andriyash, I. A., Godfrey, B. B. \& Vay, J. L. A spectral, quasi-cylindrical and dispersion-free Particle-In-Cell algorithm. Computer Physics Communications 203, 66-82 (2016).

49. Ge, Z. Y. et al. Enhancement of electron injection in laser wakefield acceleration using auxiliary interfering pulses. New Journal of Physics 14, 103015 (2012).

50. Esarey, E., Schroeder, C. B. \& Leemans, W. P. Physics of laser-driven plasma-based electron accelerators. Reviews of Modern Physics 81, 1229 (2009).

51. DOI: $10.15129 / 8301 b 69 c-8 a 57-40 e 1-a 29 d-3 f 8 d 5832 f 316$ (2021).

\section{Data availability}

Data are openly available online from the University of Strathclyde KnowledgeBase ${ }^{51}$.

\section{Acknowledgments}

The authors thank George K. Holt for valuable discussions. This work is financially supported by the Council of Scientific and Industrial Research (file number 09/045(1510)2017-EMR-I) and Department of Science and Technology, Govt. of India. We also acknowledge support from the U.K. EPSRC (grant number EP/N028694/1) and received funding from the European Union's Horizon 2020 research and innovation programme under grant agreement no. 871124 Laserlab-Europe. SRY and BE acknowledge support from the STFC (grant number ST/G008248/1). Results were obtained using the ARCHIE-WeSt High Performance Computer (www.archie-west.ac.uk) based at the University of Strathclyde, and the facilities of the N8 Centre of Excellence in Computationally Intensive Research (N8 CIR) provided and funded by the N8 research partnership and EPSRC (grant number EP/T022167/1), co-ordinated by the Universities of Durham, Manchester and York.

\section{Author contributions}

The project was conceived by DNG and AJ. Simulations were performed by SRY. Discussion, analysis, interpretation, writing and validation was undertaken by DNG, SRY, AJ, BE and DAJ. DNG, SRY and AJ contributed equally.

\section{Competing interests}

The authors declare no competing interests. 


\section{Supplementary Files}

This is a list of supplementary files associated with this preprint. Click to download.

- GuptadpLWFA2021Supplementary.pdf 